\title{
Análisis de los procesos grupales en función del género en un contexto deportivo semiprofesional
}

\author{
Analysis of the group processes regarding sex in \\ the semiprofessional sport context
}

\section{Analises dos processos grupais em função do gênero em um contexto esportivo semiprofissional}

\author{
Inmaculada González-Ponce, Francisco Miguel Leo, David Sánchez-Oliva,
} Diana Amado y Tomás García-Calvo

Universidad de Extremadura

\begin{abstract}
Resumen: El objetivo de este estudio fue analizar las diferencias de género existentes en relación al clima motivacional de los compańeros y el entrenador, la cohesión y la eficacia colectiva. Los participantes fueron 75 jugadores de fútbol de tercera división masculina y 69 jugadoras de segunda división femenina, con edades comprendidas entre los 15 y 36 ańos. Los resultados revelaron que los equipos femeninos obtuvieron valores más elevados en la cohesión social que los equipos masculinos, mientras que los equipos masculinos percibieron un mayor clima con implicación al ego entre los compańeros en comparación con los equipos femeninos. Por otro lado, tanto en equipos masculinos como femeninos, la eficacia colectiva se relacionó con la cohesión y el clima que implica a la tarea de los compañeros y el entrenador. Además, la cohesión a la tarea se mostró como el mayor predictor de la eficacia colectiva. Finalmente, se discuten los resultados y se exponen las principales conclusiones del estudio.

Palabras claves: Clima motivacional, cohesión, eficacia colectiva, fútbol, género.

Abstract: The aim of this study was to examine sex differences regarding peers and coaches motivational climates, cohesion and collective efficacy. Participants were 75 male football players from the Third Division and 69 female football players from the Second Female Division, ranging in age from 15 to 36 years old. Results showed that female teams had greater scores in social cohesion than male teams, whereas male teams perceived hig-
\end{abstract}

her peer performance climate than female teams. Furthermore, either both male and female teams, collective efficacy was related to cohesion and peers and coaches mastery climate. Besides, task cohesion was the strongest predictor of collective efficacy. Finally, results are discussed and main conclusions of the study are showed.

Keywords: Motivational climate, cohesion, collective efficacy, football, gender.

Resumo: O objetivo desse estudo é de analisar as diferenças de gênero existentes em relação ao clima motivacional dos companheiros e treinadores, a coesão e a eficácia coletiva. Participaram 75 jogadores de futebol de tercera divisão masculina e 69 jogadoras de segunda divisão femenina, com idades compreendidas entre 15 e 36 anos. Os resultados revelaram que as equipes femininas obtiveram os valores mais elevados na coesão social que as equipes masculinas, enquanto que as equipes masculinas mostraram um maior clima referente ao ego entre os companheiros em comparação com as equipes femeninas. No obstante, tantos em nas equipes masculinas como nas femeninas, a eficácia coletiva relacionou-se com a coesão e o clima que implica a tarefa dos companheiros e do treinador. Ademais, a coesão da tarefa mostrou-se com maior previsor de eficácia coletiva. Finalmente, discutemse os resultados e expóem as principais conclusóes do estudo.

Palavras Chave: Clima motivacional, coesão, eficácia coletiva, futebol, gênero.

\section{Introducción}

La finalidad que se ha perseguido con el tratamiento de los procesos grupales en el deporte ha sido optimizar el rendimiento del equipo (Heuzé, Raimbault, y Fontayne, 2006; Leo, García-Calvo, Parejo, Sánchez-Miguel, y Sánchez-Oliva, 2010). Una de las figuras que puede tratar de maximizar ese rendimiento es el entrenador (Turman, 2003; Vargas-Tonsing, Warners, y Feltz, 2003). De esta manera, uno de los

Dirección de Correspondencia: Inmaculada González Ponce. Facultad de Ciencias del Deporte, Universidad de Extremadura, Avda. de la Universidad no s/n 10071, Cáceres, Espańa. 927 257460.ingopo@unex.es objetivos prioritarios de los técnicos es ser capaz de obtener la mayor eficacia de cada uno de sus jugadores; y para ello, habría que conocer si el comportamiento de los entrenadores debería de ser el mismo en equipos femeninos y masculinos con la finalidad de conseguir el mayor rendimiento.

Por tanto, adaptarse a las características de los deportistas, no solo en cuanto a la edad o nivel de habilidad, sino también en cuanto al género, podría acercarnos a mejorar estos procesos grupales. Por ello, se puede considerar fundamental examinar las dinámicas que se desarrollan en el seno de los equipos deportivos, y establecer posibles diferencias existen- 
tes entre el género femenino y el masculino en relación a las variables grupales relevantes en el rendimiento de equipo, tales como el clima motivacional, la cohesión y la eficacia colectiva (Damato, Heard, Grove, y Eklund, 2011; Heuzé, Raimbault, et al., 2006; Leo, Sánchez-Miguel, Sánchez-Oliva, Amado, y García-Calvo, 2011).

En primer lugar, se ha visto como un clima motivacional favorable puede incidir en gran medida en la obtención de un adecuado ambiente de grupo. El término de clima motivacional, fue introducido en primer lugar por Ames $(1984$, 1992) y Ames y Archer (1998) para designar los diferentes ambientes que crean los otros significativos (padres, profesores, entrenadores, compañeros) en los entornos de logro. Dentro del ámbito del deporte en categorías sénior, el entrenador y los compañeros, son los agentes que más van a influenciar en la percepción de estos entornos del logro (Torregrosa et al., 2011; Vazou, Ntoumanis, y Duda, 2005). De este modo, el clima motivacional de los compañeros y del entrenador, entendidos como la influencia de los otros significativos sobre la motivación del jugador (Ames, 1992), puede ir encaminado bien hacia un clima motivacional que implica a la tarea, es decir, si se favorece la autonomía, se valora en gran medida el esfuerzo y el trabajo, y se enfatiza la participación; o bien, un clima motivacional que implica al ego, si se utiliza la comparación interindividual, criterios de agrupación según la capacidad, la evaluación pública, el feedback normativo y un tiempo inflexible para el aprendizaje (Balaguer, Duda, Atienza, y Mayo, 2002; Vazou et al., 2005).

De igual modo, otra de las variables grupales que se ha observado que tiene bastante relevancia dentro de los procesos grupales de los equipos deportivos ha sido la cohesión (Carron, Colman, Wheeler, y Stevens, 2002). Este término fue definido por Carron, Brawley, y Widmeyer, (1998) como "un proceso dinámico que se refleja en la tendencia de un grupo a no separarse y permanecer unido con la finalidad de conseguir sus objetivos instrumentales y/o para la satisfacción de las necesidades afectivas de sus miembros” (p. 213). En su modelo conceptual, la cohesión está formada por dos niveles claramente diferenciados, la cohesión tarea y la cohesión social. La cohesión tarea se define como el grado en que los miembros de un equipo trabajan juntos para alcanzar objetivos comunes, mientras que la cohesión social refleja el grado en que los miembros de un equipo empatizan unos con otros y disfrutan del compańerismo del grupo (Carron y Ball, 1977; Carron et al., 1998; Carron, Widmeyer, y Brawley, 1985). Además, este modelo conceptual expone cuatro antecedentes que pueden influir en la cohesión de equipo: factores ambientales, factores personales, factores de liderazgo y factores de equipo. Por lo tanto, a partir de esos factores de equipo, se va a desencadenar una serie de consecuencias que se van a dividir en dos grandes grupos; por un lado, el rendimiento individual y por otro lado el rendimiento colectivo. Dentro del rendimiento colectivo, se puede destacar la eficacia colectiva, como consecuencia importante de la cohesión de equipo.

El constructo de la eficacia colectiva fue definido por Bandura (1997) como "las creencias del grupo en el conjunto de capacidades para organizar y ejecutar las líneas de actuación requeridas para producir los logros propuestos” (p.476). Esta percepción de eficacia colectiva va a conllevar a una serie de consecuencias (metas, compromiso, satisfacción, ansiedad, rendimiento, esfuerzo y persistencia) que son el resultado de aspectos cognitivos, afectivos y comportamentales.

A pesar de que se han analizado las relaciones entre el clima motivacional, la cohesión y la eficacia colectiva tanto en hombres como en mujeres, no existen estudios específicos que comparen dichas variables en función del género. Sin embargo, el género ha demostrado ser una variable moderadora en dinámicas de grupos en investigaciones relacionados con el liderazgo (Eagly y Jonhson, 1990), la productividad (Wood, 1987) y la orientación hacia la competencia frente a la cooperación (Duda, 1987). Sí es cierto que Moreno, Cervelló, y González-Cutre (2008) mostraron cómo los hombres percibían un clima motivacional que implica al ego mayor que las mujeres, mientras que éstas percibían un clima motivacional que implica en mayor medida a la tarea que en el caso de los hombres. En cuanto a la cohesión, no hay una base teórica o empírica para predecir que los equipos femeninos y masculinos difieran en la medida en que la cohesión se asocia con el rendimiento. Sin embargo, Carron et al. (2002) apreciaron como la relación entre cohesión y rendimiento deportivo era mayor en mujeres que en hombres.

En relación a las variables de este estudio, existe un gran número de trabajos que han indagado acerca de la relación entre el clima motivacional, la cohesión y la eficacia colectiva. En primer lugar, las investigaciones existentes en relación al clima motivacional y la cohesión muestran una relación positiva entre el clima que implica a la tarea y la cohesión (Balaguer, Castillo, Moreno, Garrigues, y Soriano, 2004; GarcíaCalvo, 2006). De igual modo, las investigaciones también evidencian una relación negativa entre el clima que implica al ego y la cohesión (Balaguer, Castillo, y Duda, 2003; GarcíaCalvo, 2006). Además, Balaguer y cols., concluyeron que el clima motivacional que implica a la tarea actúa como el mejor predictor de la cohesión tanto social como tarea. En segundo lugar, en relación a las variables eficacia colectiva y clima motivacional, Heuzé, Sarrazin, Masiero, Raimbault, y Thomas (2006) demostraron en equipos femeninos que un clima motivacional que implicaba a la tarea obtiene mejores resultados en relación a la eficacia colectiva, mientras que la percepción de bajos niveles de un clima que implicaba al ego estaba asociado a una mayor percepción de eficacia colectiva al final de temporada (Leo, Sánchez-Miguel, Sánchez-Oliva, et al., 2011b). En cuanto a la relación entre la cohesión y la eficacia colectiva se ha demostrado que los jugadores que 
perciben mayor cohesión dentro de sus equipos desarrollan percepciones más fuertes sobre la eficacia colectiva del grupo (Heuzé, Sarrazin, et al., 2006; Heuzé, Raimbault, et al., 2006; Leo et al., 2010; Ramzaninezhad, Keshtan, Shahamat, y Kordshooli, 2009). Aunque existe una diferencia entre los autores que defienden que son los aspectos tarea de la cohesión los que más relación tienen con la eficacia colectiva percibida (Damato et al., 2011; Kozub y McDonnell, 2000; Paskevich, Brawley, Dorsch, y Widmeyer, 1999) mientras que otros autores defienden que ambas dimensiones, social y tarea, presentan un estrecha relación con la eficacia colectiva (Heuzé, Raimbault, et al., 2006; Spink, 1990).

Una vez analizados los constructos de nuestra investigación y observado que no existen investigaciones que relacionen las variables en función del género, el principal objetivo de este estudio es conocer las diferencias de género existentes en relación al clima motivacional, la cohesión grupal y la eficacia colectiva. Por ello, la primera hipótesis afirma que los equipos femeninos obtendrán mejores resultados en cohesión social que los equipos masculinos, mientras que los equipos masculinos conseguirán mejores resultados en cohesión tarea y en eficacia colectiva (Reis y Jelsma, 1980). La segunda hipótesis postula la existencia de una relación positiva entre el clima motivacional que implica a la tarea del entrenador y los compañeros con la cohesión tarea y social y con la eficacia colectiva, tanto en equipos masculinos como femeninos (Heuzé, Sarrazin, et al., 2006). Además, como tercera hipótesis se plantea que la cohesión tarea sera el mejor predictor de la eficacia colectiva tanto en equipos masculinos como femeninos (Kozub y McDonnell, 2000; Leo et al., 2010).

\section{Método}

\section{Participantes}

Este estudio se realizó con 144 participantes, de los cuales 75 fueron jugadores de fútbol con edades comprendidas entre los 15 y 36 años $(M=24.29 ; D T=4.37)$ y 69 jugadoras de fútbol con edades comprendidas entre los 15 y los 33 años $(M=20.01 ; D T=4.9)$ todos ellos pertenecientes a equipos de fútbol Extremeño. Los 75 jugadores pertenecían a cinco equipos del Grupo XIV de Tercera División masculina mientras que las 69 jugadoras pertenecían a cinco equipos de Segunda División femenina. Los cinco equipos seleccionados por categoría tienen similares características presupuestarias, equivalentes días de entrenamiento, instalaciones parejas y el nivel de habilidad entre jugadores y jugadoras también es análogo en los diferentes equipos seleccionados.

\section{Instrumentos}

Clima motivacional de los entrenadores. Se utilizó la adaptación al castellano del cuestionario clima motivacional percibido en el deporte (PMCSQ-2: Newton, Duda, y Yin, 2000) desarrollado por Balaguer, Guivernau, Duda, y Crespo (1997). Está formado por 24 ítems divididos en dos factores. Por un lado el clima que implica a la tarea (12 ítems, ej.: en tu grupo de entrenamiento el entrenador ayuda a mejorar a los jugadores en los aspectos en los que no son buenos) y el clima que implica al ego (12 ítems, ej.: en tu grupo de entrenamiento el entrenador anima a que los jugadores compitan entre sí). En este estudio se analizó la consistencia interna a través del coeficiente alfa de Cronbach obteniendo unos valores de .86 para el clima motivacional del entrenador que implica al ego y un .87 para el clima motivacional del entrenador que implica a la tarea.

Clima motivacional percibido en los iguales. Se empleó la versión validada al castellano del Peer Motivational Climate in Youth Sport Questionaire (PEERMCYSQ: Ntoumanis y Vazou, 2005) desarrollado por Moreno et al. (2011). Dicho instrumento consta de 19 ítems divididos en tres factores: clima tarea, competición/habilidad y conflicto. Para esta investigación, se empleó el factor de clima tarea (11 ítems, ej.: "En tu grupo de entrenamiento la mayoría de los compañeros/as se ayudan unos a otros a que se mejore") y el factor de competencia/ habilidad, denominado como clima que implica al ego (4 ítems, ej.: En tu grupo de entrenamiento se tiene más en cuenta la opinión de los compañeros/as más habilidosos/ as). Los valores de consistencia interna obtenidos fueron de .72 para el clima motivacional de los compañeros que implica al ego y de .92 para el clima motivacional de los compañeros que implica a la tarea.

Eficacia colectiva. Para valorar la eficacia colectiva se ha utilizado el instrumento diseńado por Leo, Sánchez-Miguel, Sáchez-Oliva, Amado, y García-Calvo (2011) siguiendo las propuestas realizadas por Heuzé, Raimbault, et al. (2006). El instrumento parte de una frase introductoria (ej.: "La confianza de nuestro equipo en nuestra capacidad...") y un total de 26 ítems que hacía referencia a resolver ciertas situaciones en el fútbol en ataque (13 ítems, ej.: Para mantener la posesión del balón ante presión rival) y en defensa (13 ítems, ej.: Para defender situaciones de balón parado). Las respuestas obtenidas estaban dentro de una escala tipo likert de 1 a 5 puntos, donde 1 hacía referencia a "mala" y 5 a "excelente". La estructura factorial de los 26 ítems fue analizada empleando un análisis factorial exploratorio, donde los resultados indicaron que los 26 ítems se agruparon positivamente en un único factor. Los valores de consistencia interna obtenidos fueron de .92

Cohesión de equipo. Para valorar la cohesión se ha utilizado una adaptación en castellano del Group Enviroment 
Questionary (GEQ: Carron et al., 1985) utilizada por García-Calvo (2006). Este instrumento consta de 12 ítems que están agrupados en dos factores, cohesión social (6 ítems, ej.: A los jugadores de este equipo les gustaría juntarse algunas veces cuando finalice la temporada) y cohesión tarea (6 ítems, ej.: Todos los jugadores asumen la responsabilidad ante un mal resultado del equipo). El formato de respuesta empleado es de tipo Likert con un rango de respuesta de 1 a 5 , donde 1 corresponde a totalmente desacuerdo y 5 corresponde a totalmente de acuerdo. Los valores de consistencia interna obtenidos a través del alfa de Cronbach fueron de .83 para la cohesión social y de .84 para la cohesión tarea.

\section{Procedimiento}

Para llevar a cabo la recogida de los datos se desarrolló un protocolo de actuación para asegurar que la obtención de los datos fuera similar en todos los participantes implicados en la investigación. En primer lugar, se contactó con los entrenadores de los diferentes equipos para solicitarles la inclusión de sus equipos dentro del estudio. A su vez, se le informó acerca de los objetivos y procedimientos del estudio que se llevaría a cabo en caso de su disponibilidad para participar en la investigación. Posteriormente, los jugadores/as también fueron informados acerca de los objetivos de la investigación, y se les comentó que su participación era voluntaria y las respuestas realizadas serían tratadas confidencialmente. Así mismo, los padres de las jugadoras menores de edad tuvieron que firmar un consentimiento informado con la finalidad de que pudieran realizar el cuestionario como el resto de sus compañeras. Dichos cuestionarios fueron rellenados por los participantes en el vestuario antes del entrenamiento, sin la presencia del entrenador, de manera individual y en un clima adecuado. El proceso de realización de los cuestionarios duró aproximadamente 25 minutos. El investigador principal estuvo presente en el momento en que los sujetos rellenaban los cuestionarios y reiteró en la posibilidad de aclarar cualquier tipo de duda que surgiese durante el proceso. La medición se realizó tres jornadas antes de finalizar la temporada deportiva por lo que los jugadores tenían una visión completa de la percepción de cada una de las variables estudiadas.

\section{Análisis de datos}

Para el tratamiento de los datos se ha utilizado el software SPSS 18.0, a través del cual se han realizado diferentes tipos de análisis para buscar las relaciones existentes entre las distintas variables en función del género. Las técnicas utilizadas han sido el análisis de fiabilidad de las escalas, el análisis descriptivo de cada variable, la prueba T- Student, la estadística correlacional de Pearson para establecer la relación entre cada una de las variables y el análisis de regresión lineal para conocer el mejor predictor de la eficacia colectiva.

\section{Resultados}

Estadísticos descriptivos y comparación de medias

En primer lugar, se analizan los estadísticos descriptivos de cada una de las variables medidas a través de los instrumentos de la investigación. En la Tabla 1 se puede apreciar de forma significativa como los hombres perciben un mayor clima motivacional de los compañeros que implica al ego que las mujeres. Respecto a la cohesión, se observó que la media de los diferentes factores presentaban puntuaciones moderadamente altas, siendo los equipos femeninos los que obtuvieron mayor cohesión social que los equipos masculinos apreciándose diferencias significativas. En cambio, son los hombres los que mostraron mayor cohesión a la tarea y eficacia colectiva con respecto a las mujeres aunque no de forma significativa.

Tabla 1. Análisis descriptivos y comparación de Medias

\begin{tabular}{lllll}
\hline & \multicolumn{3}{c}{$M \pm D T$} & \multirow{2}{*}{$t$} \\
\cline { 2 - 4 } & Hombres & Mujeres & & \multirow{2}{*}{$p$} \\
\hline Clima Compañeros Ego & $3.48 \pm .62$ & $2.95 \pm .85$ & 4.26 & .00 \\
Clima Compañeros Tarea & $3.97 \pm .78$ & $3.94 \pm .64$ & -.54 & .58 \\
Clima Entrenador Ego & $2.56 \pm .92$ & $2.78 \pm .99$ & -1.33 & .18 \\
Clima Entrenador Tarea & $3.71 \pm .86$ & $3.54 \pm .93$ & 1.11 & .26 \\
Cohesión Social & $3.82 \pm .62$ & $4.08 \pm .84$ & -2.14 & .03 \\
Cohesión Tarea & $3.63 \pm .68$ & $3.45 \pm .89$ & 1.39 & .16 \\
Eficacia Colectiva & $3.41 \pm .86$ & $3.29 \pm .62$ & 1.19 & .23 \\
\hline
\end{tabular}

\section{Correlaciones}

Con el objetivo de comprobar las relaciones existentes entre los diferentes factores de la investigación, se decidió llevar a cabo un análisis de correlaciones bivariadas. En la Tabla 2, se puede ver las relaciones entre el clima motivacional del entrenador y los compañeros, la cohesión grupal y la eficacia colectiva. Referente a la cohesión social se observó la asociación de forma positiva y significativa con el clima motivacional de los compańeros que implica al ego, pero solo en equipos masculinos. También existió una correlación de forma positiva y significativa entre la cohesión social con el clima motivacional de los compañeros y el entrenador que implica a la tarea tanto en equipos masculinos como femeninos.

Por su parte, la cohesión tarea se relacionó de forma positiva con el clima motivacional de los compañeros que implica al ego y a la tarea tanto en equipos masculinos como femeninos. Destacar también como la cohesión tarea se correlacionó de forma negativa y significativa con el clima motivacional del entrenador que implica al ego pero solo en equipos femeninos.

Por último, la eficacia colectiva, se relacionó de forma po- 
sitiva y significativa con el clima motivacional de los compañeros y el entrenador que implica a la tarea tanto en equipos masculinos como femeninos. También existió una asociación positiva y significativa entre la eficacia colectiva y el clima motivacional de los compañeros que implica al ego tanto en equipos masculinos como femeninos. De igual forma, la eficacia colectiva se correlacionó de forma significativa y positiva con la cohesión social y la cohesión tarea tanto en equipos femeninos como masculinos.

Tabla 2. Correlaciones entre los factores del estudio

\begin{tabular}{|c|c|c|c|c|c|c|c|c|c|c|c|c|}
\hline & 1 & & 2 & & 3 & & 4 & & 5 & & 6 & \\
\hline & $\mathrm{H}$ & M & $\mathrm{H}$ & M & $\mathrm{H}$ & M & $\mathrm{H}$ & M & $\mathrm{H}$ & $\mathrm{M}$ & $\mathrm{H}$ & M \\
\hline 1. C. Compañeros Ego & - & - & & & & & & & & & & \\
\hline 2. C. Compañeros Tarea & $.40^{* *}$ & -.17 & & & & & & & & & & \\
\hline 3. C. Entrenador Ego & $.34^{* *}$ & $.41^{* *}$ & -.13 & -.12 & & & & & & & & \\
\hline 4. C. Entrenador Tarea & $.25^{*}$ & .15 & $.72^{* *}$ & $.65^{* *}$ & $.34^{* *}$ & $.43^{* *}$ & & & & & & \\
\hline 5. Cohesión Social & $.43^{* *}$ & .16 & $.54^{* *}$ & $.59^{* *}$ & -.03 & -.06 & $.51^{* *}$ & $.43^{* *}$ & & & & \\
\hline 6. Cohesión Tarea & $.29^{* *}$ & $.29^{*}$ & $.66^{* *}$ & $.59^{* *}$ & -.19 & $-.24^{*}$ & $.64^{* *}$ & $.75^{* *}$ & $.54^{* *}$ & $.59^{* *}$ & & \\
\hline 7. Eficacia Colectiva & $.32^{* *}$ & $.35^{* *}$ & $.68^{* *}$ & $.48^{* *}$ & -.15 & -.17 & $.62^{* *}$ & $.71^{* *}$ & $.45^{* *}$ & $.35^{* *}$ & $.73^{* *}$ & $.64^{* *}$ \\
\hline
\end{tabular}
${ }^{* *} p<.01 ;{ }^{*} p<.05$

\section{Análisis de regresión lineal}

En la Tabla 3, se muestran los principales estadísticos obtenidos al realizar un análisis de regresión por pasos utilizando como variable dependiente la eficacia colectiva. Como se aprecia en los resultados, se obtuvo como mayor predictor la cohesión tarea tanto en equipos masculinos como femeninos. En equipos masculinos, el porcentaje de varianza explicada es del $53 \%$ mientras que en los equipos femeninos es similar con un 52\%. En ambos casos, dicha variable predijo de forma positiva, es decir, cuanta mayor cohesión hacia la tarea fue percibida por los jugadores, mayor fue la percepción de eficacia colectiva en el equipo. En el segundo paso, en equipos masculinos aparece como predictor de la eficacia colectiva el clima motivacional que implica a la tarea de los compañeros con un $7 \%$ de varianza explicada, mientras que en equipos femeninos es el clima motivacional del entrenador que implica a la tarea con un 6\% de la varianza explicada; al igual que la anterior predice de forma positiva, pero la capacidad predictiva en este caso es muy débil. En el tercer paso, el clima motivacional que implica al ego de los compañeros solamente predice en los equipos femeninos con un 3\% de varianza explicada; al igual que la anterior predice de forma positiva, pero la capacidad predictiva es muy débil y por tanto hay que tratar los resultados con cautela.
Tabla 3. Coeficientes del análisis de regresión por pasos considerando como variable dependiente la Eficacia Colectiva.

\begin{tabular}{|c|c|c|c|c|}
\hline \multicolumn{5}{|c|}{ Equipos Masculinos } \\
\hline Variable & $\beta$ & $R^{2}$ & $t$ & $P$ \\
\hline Paso 1 & & .53 & & \\
\hline Cohesión Tarea & .73 & & 9.13 & .00 \\
\hline Paso 2 & & .60 & & \\
\hline Cohesión Tarea & .49 & & 5.01 & .00 \\
\hline Clima Compañeros Tarea & .35 & & 3.53 & .00 \\
\hline \multicolumn{5}{|c|}{ Equipos Femeninos } \\
\hline Variables & $\beta$ & $R^{2}$ & $t$ & $p$ \\
\hline Paso 1 & & .52 & & \\
\hline Cohesión Tarea & .72 & & 8.61 & .00 \\
\hline Paso 2 & & .58 & & \\
\hline Cohesión Tarea & .43 & & 3.62 & .00 \\
\hline Clima Entrenador Tarea & .38 & & 3.18 & .00 \\
\hline Paso 3 & & .61 & & \\
\hline Cohesión Tarea & .36 & & 2.96 & .00 \\
\hline Clima Entrenador Tarea & .41 & & 3.50 & .00 \\
\hline Clima Compañeros Ego & .18 & & 2.18 & .03 \\
\hline
\end{tabular}

\section{Discusión}

Tras el análisis de los resultados y teniendo en cuenta que el objetivo principal de este estudio era conocer las diferencias de género existentes en relación al clima motivacional, la cohesión grupal y la eficacia colectiva, se procedió a realizar la discusión de los resultados a partir de las hipótesis del estudio. 
Así, la primera de las hipótesis enunciaba que los equipos femeninos obtendrían mayor cohesión social que los equipos masculinos, mientras que los equipos masculinos conseguirían mejores resultados en cohesión tarea y en eficacia colectiva. En primer lugar, se puede destacar como los equipos femeninos lograron puntuaciones más altas que los equipos masculinos en relación a la variable cohesión social. Anteriormente, Reis y Jelsma (1980) ya habían demostrado puntuaciones más elevadas de las mujeres en las relaciones interpersonales con las compañeras de equipo que los hombres. En cambio, son los hombres los que presentaban valores más elevados de cohesión tarea y eficacia colectiva que las mujeres aunque no de forma significativa, esto puede ser debido a la mayor competitividad que estos muestra en comparación con las chicas donde el aspecto cooperativo y de ocio está más asimilado a la hora de competir (Torregrosa et al., 2011). Por tanto, a partir de los resultados anteriormente comentados, se puede afirmar la primera hipótesis, confirmando que las jugadoras de los equipos femeninos percibían mayores niveles respecto a la cohesión social que los jugadores de equipos masculinos, por otra parte los equipos masculinos obtuvieron valores sensiblemente más altos en cohesión tarea y eficacia colectiva, aunque no de forma significativa.

Siguiendo con la discusión de los resultados, se analiza la segunda de las hipótesis planteadas, la cual enunciaba la existencia de una relación positiva entre el clima motivacional del entrenador y los compañeros que implica a la tarea, la cohesión tarea y social y la eficacia colectiva, tanto en equipos masculinos como femeninos. En este sentido, el clima motivacional del entrenador que implica a la tarea se relacionaba de forma positiva y significativa con la cohesión y la eficacia colectiva. Estos resultados ya habían sido encontrados anteriormente por otros autores como Balaguer et al. (2004) quienes seńalaban que los jugadores y jugadoras que percibían un clima motivacional que implicaba a la tarea en el ambiente de entrenamiento mostraban mayores niveles de cohesión en sus equipos.

En relación al clima motivacional y la eficacia colectiva Leo y cols. (2011) encontraron que el clima que implica a la tarea de los compańeros y el entrenador predicen de forma positiva la eficacia colectiva. Igualmente, Heuzé, Sarrazin, et al. (2006) con jugadores de balonmano femenino, encontró relaciones positivas y significativas entre el clima motivacional que implica a la tarea, la cohesión y la eficacia colectiva. Así mismo, Damato et al. (2011) demostraron que aquellos jugadores de fútbol con altos niveles de eficacia colectiva obtenían altos niveles de cohesión hacia la tarea y percibían climas más implicados hacia la tarea. Finalmente también existía una relación de forma significativa y positiva entre la cohesión y la eficacia colectiva. De este modo, atendiendo a los resultados encontrados y tras la discusión expuesta anteriormente, se puede afirmar la segunda hipótesis, estableciendo que existe una relación directa entre el clima que implica a la tarea por parte del entrenador y los compañeros, la cohesión de equipo y la eficacia colectiva.

Finalmente, la tercera hipótesis postulaba que la cohesión tarea sería el mejor predictor de la eficacia colectiva tanto en equipos masculinos como femeninos. En concordancia con la hipótesis, los resultados mostraban como en esta investigación, la cohesión tarea fue el mayor predictor de la eficacia colectiva tanto en equipos masculinos como femeninos. Estos resultados coinciden con el grupo de investigadores que consideraban la cohesión de grupo como un antecedente de la eficacia colectiva, como es el caso de Kozub y McDonnell (2000), donde en su estudio con equipos de rugby profesionales, afirmaban que los jugadores que percibían en sus equipos una alta cohesión a la tarea tendían a mostrar en sus equipos mayores niveles de eficacia colectiva. Igualmente, Leo et al. (2010) con jugadores de baloncesto, encontraron que la cohesión de equipo fue el principal predictor de la eficacia colectiva. Por tanto, una vez expuesto los resultados se puede afirmar que la tercera hipótesis se cumple, es decir, que el mayor predictor de la eficacia colectiva fue la cohesión tarea.

Una vez observado que existían diferencias significativas entre hombres y mujeres en relación a las variables del estudio, sería positivo seguir con esta línea de investigación. Para ello, podría resultar conveniente seleccionar una muestra más profesionalizada en este contexto tanto en equipos masculinos como femeninos para futuras investigaciones, ya que tendrán mayor repercusión en el alto rendimiento. De igual modo, se podría realizar varias mediciones a lo largo de la temporada, para ver la fluctuación que sufren las variables del estudio durante todo el periodo deportivo.

De esta manera, la principal conclusión que se puede extraer de esta investigación es que las diferencias significativas existentes entre hombres y mujeres en relación a las variables cohesión social y clima motivacional que implica al ego, deberían servir para realizar un enfoque distinto en función del género del equipo al cual se entrene. Por lo tanto, según este estudio los entrenadores ya no solo tendrán que tener en cuenta aspectos como la edad, la habilidad del grupo, la experiencia, etc., sino que también el género puede ser una variable muy importante que puede influir de forma diferente en el desarrollo de los procesos grupales. A pesar de ello, los resultados también muestran que sería interesante desarrollar, tanto en equipos femeninos como masculinos, actividades durante los entrenamientos focalizados en los aspectos tarea de la cohesión, ya que éste ha sido el mayor predictor de la eficacia colectiva. 


\section{Referencias bibliográficas}

1. Ames, C. (1984). Competitive, cooperative, and individualistic goal structures: A cognitive-motivational analysis. En R. Ames y C. Ames (Eds.), Research on motivation in education: Vol.1. Student motivation (pp. 177-208). New York: Academic Press.

2. Ames, C. (1992). Achievement goals, motivational climate, and motivational processes. En G. C. Roberts (Ed.), Motivation in sport and exercise (pp. 161-176). Champaign, IL: Human Kinetics.

3. Ames, C., y Archer, J. (1988). Achievement goals in the classroom: Student's learning strategies a motivation processes. Journal of Educational Psychology, 80, 260-267.

4. Balaguer, I., Castillo, I., y Duda, J. L. (2003). Interrelaciones entre el clima motivacional y la cohesión en futbolistas cadetes. Edupsykhé, 2, 243-258.

5. Balaguer, I., Castillo, I., Moreno, Y., Garrigues, V., y Soriano, L. (2004). El clima motivacional y la cohesión en equipos de fútbol. Encuentros en Psicología Social, 2, 152-156.

6. Balaguer, I., Duda, J. L., Atienza, F. L., y Mayo, C. (2002). Situational and dispositional goals as predictors of perceptions of individual and team improvement, satisfaction and coach ratings among elite female handball teams. Psychology of Sport and Exercise, 3, 293-308. doi:10.1016/S1469-0292(01)00025-5

7. Balaguer, I., Guivernau, M., Duda, J. L., y Crespo, M. (1997). Análisis de la validez de constructo y de la validez predictiva del cuestionario de clima motivacional percibido en el deporte (PMCSQ-2) con tenistas españoles de competición. Revista de Psicología del Deporte, 11, 41-57.

8. Bandura, A. (1997). Self-efficacy: The exercise of control. New York: Freeman.

9. Carron, A. V., y Ball, J. R. (1977). Cause-effect characteristics of cohesiveness and participation motivation in intercollegiate hockey. International Review of Sport Sociology, 12, 49-60.

10. Carron, A. V., Brawley, L. R., y Widmeyer, W. N. (1998). The measurement of cohesiveness in sport groups. En J. L. Duda (Ed.), Advances in sport and exercise psychology measurement (pp. 213-226). Morgantown, WV: Fitness Information Technology.

11. Carron, A. V., Colman, M. M., Wheeler, J., y Stevens, D. (2002). Cohesion and performance in sport a meta analysis. Journal of Sport y Exercise Pshycology, 24, 168-188.

12. Carron, A. V., Widmeyer, W. N., y Brawley, L. R. (1985). The development of an instrument to assess cohesion in sport teams: The Group Environment Questionnaire. Journal of Sport Psychology, 7, 244-266.

13. Damato, G., Heard, P., Grove, J. R., y Eklund, R. C. (2011). Multivariate relationships among efficacy, cohesion, self- talk and motivational climate in elite sport. Pamukkale Journal of Sport Sciences, 2, 6-26.

14. Duda, J. (1987). Toward a developmental theory of children's motivation in sport. Journal of Sport and Exercise Psychology, 9, 130-145.

15. Eagly, A. H., y Jonhson, B. T. (1990). Gender and leadership style: A meta-analysis. Psychological Bulletin, 108, 233-256.

16. García-Calvo, T. (2006). Motivación y comportamientos adaptativos en jóvenes futbolistas. Universidad de Extremadura.

17. Heuzé, J. P., Sarrazin, P., Masiero, M., Raimbault, N., y Thomas, J. P. (2006). The relationships of perceived motivational climate to cohesion and collective efficacy in elite female teams. Journal of Applied Sport Psychology, 18, 201-218. doi:10.1080/10413200600830273

18. Heuzé, Raimbault, N., y Fontayne, P. (2006). Relationships between cohesion, collective efficacy and performance in professional basketball teams: an examination of mediating effects. Journal of sports sciences, 24, 59-68. doi:10.1080/02640410500127736

19. Kozub, S. A., y McDonnell, J. F. (2000). Exploring the relationship between cohesion and collective efficacy in rugby teams. Journal of Sport Behavior, 23, 120-129.
20. Leo, F. M., García-Calvo, T., Parejo, I., Sánchez-Miguel, P. A., y Sánchez-Oliva, D. (2010). Interacción de la cohesión en la eficacia percibida, las expectativas de éxito y el rendimiento en equipos de baloncesto. Revista de Psicología del Deporte, 19, 89-102.

21. Leo, F. M., Sánchez-Miguel, P. A., Sáchez-Oliva, D., Amado, D., y García-Calvo, T. (2011). Incidencia de la cooperación, la cohesión y la eficacia colectiva en el rendimiento en equipos de fútbol. Revista Internacional de Ciencias del Deporte, 7, 341-354. doi:10.5232/ricyde2011.02601

22. Leo, F. M., Sánchez-Miguel, P. A., Sánchez-Oliva, D., Amado, D., y García-Calvo, T. (2011a). Análisis del clima motivacional como antecedente de la eficacia colectiva en futbolistas semiprofesionales. Revista de Psicología del Deporte, 21, 159-162.

23. Leo, F. M., Sánchez-Miguel, P. A., Sánchez-Oliva, D., Amado, D., y García-Calvo, T. (2011b). Análisis de las relaciones del clima motivacional y la cohesión de equipo en el grado de compromiso de jóvenes futbolistas. Cultura, Ciencia y Deporte, 6, 5-13.

24. Moreno, J. A., Cervelló, E., y González-Cutre, D. (2008). Relationships among goal orientations, motivational climate and flow in adolescent athletes: differences by gender. The Spanish journal of psychology, 11, 181-91.

25. Moreno, J. A., Conte, L., Martínez, G. C., Alonso, N., González-Cutre, D., y Cervelló, E. (2011). Propiedades psicométricas del Peer Motivational Climate in Youth Sport Questionnaire (PeerMCYSQ) con una muestra de deportistas espańoles. Revista de Psicología del Deporte, 20, $101-118$.

26. Newton, M. L., Duda, J. L., y Yin, Z. (2000). Examination of the psychometric properties of the perceived Motivational Climate in Sport Questionnaire-2 in a simple of female athletes. Journal of Sport Stience, $18,275-290$

27. Paskevich, D. M., Brawley, L. R., Dorsch, K. D., y Widmeyer, W. N. (1999). Relationship between collective efficacy and cohesion: Conceptual and measurement issues. Group Dynamics: Theory, Research, and Practice, 3, 210-222.

28. Ramzaninezhad, R., Keshtan, M. H., Shahamat, M. D., y Kordshooli, S. S. (2009). The relationship between collective efficacy, group cohesion and team performance in professional. Brazilian Journal of Biomotricity, 3, 31-39.

29. Reis, H., y Jelsma, B. (1980). A social psychology of sex differences in sport. En W. F. Straub (Ed.), Sport psychology. NewYork: Ithaca.

30. Spink, K. S. (1990). Group cohesion and collective efficacy of volleyball teams. Journal of Sport y Exercise Pshycology, 12, 301-311.

31. Torregrosa, M., Viladrich, C., Ramis, Y., Azócar, F., Latinjak, A. T., y Cruz, J. (2011). Efectos en la percepción del clima motivacional generado por los entrenadores y compañeros sobre la diversión y el compromiso. Diferencias en función de género. Revista de Psicología del Deporte, 20, 243-255.

32. Turman, P. D. (2003). Coaches and cohesion:The Impact of coaching techniques on team cohesion in the small group sport setting. Journal of Sport BehaviorJournal of Sport Behavior, 26, 86-104.

33. Vargas-Tonsing, T. M., Warners, A. L., y Feltz, D. L. (2003). The predictability of coaching efficacy on team efficacy and player efficacy in volleyball. Journal of Sport Behavior, 26, 396-407.

34. Vazou, S., Ntoumanis, N., y Duda, J. L. (2005). Predicting young athletes' motivational indices as a function of their perceptions of the coach- and peer-created climate predicting young athletes' motivational indices as a function of their perceptions of the coach- and peercreated climate. Psychology of Sport and Exercise, 7, 215-233.

35. Wood, W. (1987). Meta-analytic review of sex differences in group performance. Psychological Bulletin, 102, 53-71. 